\title{
An fMRI Study Investigating Adolescent Brain Activation by Rewards and Feedback
}

\author{
Won-Hee Choi ${ }^{1}$, Jung-Woo Son ${ }^{\bowtie}$, Yeoung-Rang Kim² ${ }^{2}$, Jong-Hyun Oh${ }^{1}$, Sang-Ick Lee', \\ Chul-Jin Shin 1 , Sie-Kyeong Kim¹, Gawon Ju1', Seungbok Lee ${ }^{3}$, Seongwoo $\mathrm{Jo}^{3}$ and Tae Hyon $\mathrm{Ha}^{4}$ \\ ${ }^{1}$ Department of Psychiatry, Chungbuk National University Hospital, Cheongju, Republic of Korea \\ 2Department of Psychiatry, Cheongju Medical Health Hospital, Cheongju, Republic of Korea \\ ${ }^{3}$ Department of Psychology, Chungbuk National University, Cheongju, Republic of Korea \\ ${ }^{4}$ Department of Psychiatry, Bundang Seoul National University Hospital, Seongnam, Republic of Korea
}

Objective This study aimed to investigate the adolescent brain activation patterns in response to performance feedback (PF), social reward (SR) and monetary reward (MR) and their association with psychological factors.

Methods Functional magnetic resonance imaging (fMRI) was performed while middle school boys $(\mathrm{n}=15)$ performed tests pertained to PF, SR and MR. The brain activation pattern in each condition was investigated, and the extent of brain activation in each of the three conditions was compared at once.

Results The caudate and the dorsal prefrontal area were activated in all three conditions. Furthermore, the cuneus showed significantly greater activation in the PF condition than the SR or MR condition. And the self - related areas, such as the right precentral gyrus and paracenral lobule, were more activated in the SR condition than the PF or MR condition. The left middle frontal gyrus was more activated in the MR condition than the PF or SR condition.

Conclusion Not only various reward stimuli but also feedback stimulus might commonly activate dorsal prefrontal and subcortical area in adolescents. Moreover, several different brain activation patterns were also observed in each condition. The results of this study could be applied to planning of learning and teaching strategy for adolescents in various ways.

Psychiatry Investig 2013;10:47-55

Key Words Adolescent, Performance feedback, Social reward, Monetary reward, fMRI.

\section{INTRODUCTION}

Of the many characteristics of adolescence, 'reward seeking behavior' is gaining much attention. ${ }^{1}$ Reward seeking helps adolescents achieve independence by increasing their desire for achievement, but can also cause an obsession with risky behaviors, instability or deviations to satisfy immediate pleasure. Drug abuse and eating disorders, which appear during adolescence, are also closely related to reward-seeking behaviors. ${ }^{2}$

Recent studies have used brain imaging to investigate reward-seeking behaviors in adolescents. Galvan et al. ${ }^{3}$ conduct-

Received: March 4, 2012 Revised: June 6, 2012

Accepted: August 16, 2012 Available online: February 8, 2013

$\triangle$ Correspondence: Jung-Woo Son, MD, PhD

Department of Psychiatry, Chungbuk National University Hospital, 776 1Sunhwan-ro, Heungdeok-gu, Cheongju 361-711, Republic of Korea

Tel: $+82-43-269-6182$, Fax: $+82-43-267-7951$

E-mail: mammosss@hanmail.net

(a) This is an Open Access article distributed under the terms of the Creative Commons Attribution Non-Commercial License (http://creativecommons.org/licenses/by$\mathrm{nc} / 3.0$ ) which permits unrestricted non-commercial use, distribution, and reproduction in any medium, provided the original work is properly cited. ed a functional magnetic resonance imaging (fMRI) study investigating the brain activation by reward stimulus in groups of normal children, adolescents and adults. This study found that adolescents show significantly lower activation of the orbitofrontal cortex, which is associated with stimulus control and the regulation of emotions, than adults, while the activation of nucleus accumbens, which is associated with reward seeking, was very close to the level of activation in adults. In contrast, a study by Bjork et $\mathrm{al}^{4}{ }^{4}$ reported that the anticipation for gaining rewards caused greater activation of the right ventral striatum and amygdala in adolescents than in young adults. Van Leijenhorst et al. ${ }^{5}$ have reported that the activation of the anterior insula was much higher in adolescents than in adults when performing a task that cannot accurately predict the extent of the reward.

The above studies all used monetary rewards. Monetary reward have also been used frequently in brain imaging studies investigating the brain activation by reward stimulus in adults. ${ }^{6,7}$ However, monetary reward is just one of the many stim- 
uli that trigger human behavior. Di Chiara and Vanentina ${ }^{8}$ have claimed that all stimuli that function as a positive reinforcement in motivating individuals could be rewards. Meanwhile, 'feedback' is considered to be something that is similar to a reward and can act as positive or negative reinforcement. Even in real life, adolescents consider other people's evaluation of their own lives important, and they also modify their current study plans through feedback on their test results. Some brain imaging studies have also used reinforcements other than monetary reward, such as social reward and performance feedback.

Social reward is comprised of social status (e.g., reputation) and social assessment (e.g., praise) and provides important motivation for an individual's decision-making and goal-oriented activities. Izuma et al. ${ }^{9}$ used monetary and social rewards (e.g., a good reputation) in an fMRI study on adults, showing that both stimuli activated the striatum. Zink et al. ${ }^{10}$ have also reported a similar result. A study that compared the differences between men and women with regards to monetary and social rewards during 'anticipation' found that the activation of the mesolimbic brain region of men was far more noticeable for monetary rewards than for social rewards, whereas women showed activation of the mesolimbic brain region in both reward conditions. ${ }^{11}$

Performance feedback refers to providing objective facts (e.g., "correct/incorrect") about the final outcome of task performance, and using this feedback can help in modifying a strategy or decision making. Tricomi et al. ${ }^{12}$ have reported that the bilateral caudate nucleus was clearly activated when performance feedback was provided during task performance. Moreover, a study by Nieuwenhuis et al. ${ }^{13}$ reported that the rostral anterior cingulate cortex, posterior cingulate cortex, right superior frontal gyrus and striatum were activated when positive feedback (rather than negative feedback) was given. A different report found that when evaluative feedback (clever/stupid), which is considered a social reward, was compared with performance feedback (correct/incorrect), the cortical midline structure was activated in response to the social reward, but no region was activated for the performance feedback. ${ }^{14}$

As mentioned above, various studies investigating reinforcements other than monetary rewards are being conducted in adults. However, our search results revealed that there are few reports that have studied the brain activation patterns for social reward and performance feedback in adolescents. In particular, no single study has investigated the brain responses to monetary rewards, social rewards and performance feedback together in adolescents.

Alternatively, reward-seeking behaviors during adolescence may also be linked to the psychological characteristics of this period in life. Depressive feelings may be one of these characteristics. Pizzagalli et al. ${ }^{15}$ claimed that depressed individuals lose their ability to integrate the reinforced experiences with the passage of time when performing a task with the likelihood of a reward, and an fMRI study by Forbes et al. ${ }^{16}$ also reported that children with major depressive disorder have a relatively reduced neurological response in the caudate nucleus and the orbitofrontal cortex in response to the social reward stimulus of a smiling picture compared to normal children. In addition, Narcissism is also one of the important factors in adolescents. It is divided into 'overt narcissism' and 'covert narcissism', in which the former appears as actively demanding praise from others and flaunting magnificence, while the latter is related to identifying other people's reactions and avoiding situations in which one might be criticized or condemned and thereby protecting oneself. ${ }^{17}$ Considering all of these facts, we believe it would be very meaningful to investigate the correlation between the adolescent brain activation patterns related to rewards or feedback and depressive feelings and narcissistic tendencies.

Therefore, we used fMRI to examine the adolescent brain responses when asked to perform tasks associated with performance feedback, social reward and monetary reward. Moreover, we wanted to identify what kinds of relationship exists between depressive feeling or covert narcissism and the brain regions associated with rewards.

\section{METHODS}

\section{Participants}

The subjects were recruited through advertisements in hospitals and schools targeting male middle school students in Cheongju city. The inclusion criteria were as follows: 1) between 13 and 15 years of age; 2) right-handedness; 3) IQ above 80 , as determined by the abbreviated IQ test; 4) no history of head trauma, convulsive disorder, or other major neurological disorders; 5) not suffering from serious medical or surgical illness and 6) not affected with psychiatric disorders, as determined by interviews using the Kiddie-Schedule for Affective Disorders and Schizophrenia-Present and Lifetime Version-Korean Version (K-SADS-PL-K). ${ }^{18}$ Ultimately, 15 subjects were analyzed.

This study obtained approval from the Bioethics Committee at the Chungbuk National University Hospital, and written consent forms were obtained after the study objectives and methods were fully explained to the study participants.

\section{Behavioral testing}

The short-form of the Wechsler Intelligence Scale for Children-Revised (WISC-R) was used to assess the intelligence of 
the study group. This assessment method included 4 subtests from the WISC-R, including arithmetic, vocabulary, sequence arrangement and block design. ${ }^{19}$

The assessment of depressive feeling was evaluated using the standardized Korean translation ${ }^{20}$ of Kovacs Children's Depression Inventory (CDI). ${ }^{21}$ The Covert Narcissism Scale (CNS) developed by Gang and Chung ${ }^{22}$ was used to determine covert narcissism. This scale, which was designed from the study by Akhtar and Thomson, ${ }^{23}$ has a total of 45 questions to be answered with the 5 point likert scale. A higher score indicates a higher tendency for covert narcissism. The CNS is divided into the following sub-factors: 1) insecurity about goals, 2) a desire for recognition/grandiose self-fantasy, 3) exploitation/egocentricity, 4) over-sensitivity/weakness and 5) timidity/lack of self-confidence. The total CNS score and each score for the $5 \mathrm{sub}$-factors were used in this study.

\section{fMRI implementation plan and data analyses}

\section{Experimental stimulus}

This study was designed with block condition. The rightleft discrimination test, which was created by adopting the task used in the study by Ghandi et al. ${ }^{24}$ was presented to the participants. Galvan ${ }^{1}$ has claimed that large differences in the task completion rates of the subjects of a brain imaging study related to rewards make it difficult to distinguish whether the brain activation results were indeed related to the rewards or whether those differences were due to success or failure in the task. Therefore, we selected a very simple task to maintain task success rate. The subjects were asked to click on the mouse button (left or right) corresponding to the location (left or right) of an abstract design that was momentarily shown on the monitor screen. E-prime software version 2.0 was used for the stimulation.

Each block corresponded to the situation with performance feedback (PF), social reward (SR), monetary reward (MR), or no reward (NR). NR block was included as a control condition. A total of 8 blocks were implemented, including the 2 blocks that corresponded to each condition. 10 trials were conducted for each block, in which an abstract design appeared on the left or right side of the screen. The abstract design was set to randomly appear 5 times on the left side and 5 times on the right side, for a total of 10 trials. In addition, 8 blocks were set to appear randomly. As a safeguard for the subjects becoming bored if continuously presented with the same stimulus, the research team itself created the abstract designs and selected a total of 5 designs to be presented twice in each block. Each design was presented once on the left side and once on the right. Thus, the condition for all of the designs seen in each block was set to be identical.
The organization of each trial was as follows: right-left discrimination stimulus (200 ms) - blank screen (1,000 ms) word related to rewards (1,300 ms) - screen where a small cross appears (500 ms). The subjects were to determine left or right with a click of the mouse button at the moment when the blank screen appeared after the right-left discrimination stimulus, and the reward-related word was presented to the subject based on the result. 30 seconds were allotted for each block, and the rest period between the blocks was 15 seconds. The total amount of time for the fMRI screening was 6 minutes and 15 seconds, including the 15 seconds of the dummy period.

For each of the 4 conditions (NR, PF, SR and MR), the reward-related words were presented as follows. This method is similar to the technique used in the study by Pan et al.: ${ }^{14}$

1) For NR, the Korean word meaning 'next' ('그다음') was continuously presented after performing the task, regardless of the correct answer.

2) For PF, the Korean word meaning 'correct' ('정답임') or 'right' ('맞았음') appeared when the task was performed correctly, while the Korean word for 'next' ('그다음') was presented when the task was performed incorrectly. If all 10 trials within one block were performed correctly, then 'correct' and 'right' would each be presented to the subject 5 times.

3) For SR, the Korean word meaning 'great' ('대단해') or 'good' ('잘했어') appeared when the task was performed correctly, while the Korean word for 'next' ('그다음') was presented when the task was performed incorrectly. If all 10 trials within one block were performed correctly, then 'great' and 'good' would each be presented to the subject 5 times.

4) For MR, the Korean word that corresponds to Korean currency [300 won ('300원') or 400 won ('400원')] appeared when the task was performed correctly, while the Korean word for 'next' ('그다음') was presented when the task was performed incorrectly. If all 10 trials within one block were performed correctly, then ' 300 won' or ' 400 won' would each be presented to the subject 5 times. 300 won is equivalent to approximately 0.267 US dollars and 400 won is equivalent to approximately 0.357 US dollars. The subjects received the amount that corresponds to the total of the correct answers as prize money. If all of the performances were correct, 14,000 won (approximately 12.50 US dollars) could be received. We used ' 300 won' and ' 400 won' to restrain the calculation of the prize money during task performance as much as possible. Therefore, we selected combinations in which mental arithmetic was not relatively easy.

All of the reward-related words were 3-syllable Korean words. The reward stimulus presented for the MR condition ('300 won', '400 won') had to include 3 numbers and 1 syllable. However, when these words are actually read in Korean, they 
are read as 3 syllables instead of 4 syllables.

All of the subjects heard the description of this experiment prior to fMRI screening and performed practice task. The description of this experiment also included the statement that the prize money would actually be given based on the results from the MR condition. After the description of the experiment, a practice task consisting of 4 blocks was completed. The prize money awarded from the results of the MR was not paid for the practice task.

\section{fMRI scanning and analyses}

The magnetic resonance images were taken using a 3.0 Tesla whole-body ISOL Technology FORTE scanner (ISOL Technology, Korea) at Daejeon KAIST. After the first fMRI scan was completed, the T1 anatomical scan was obtained. To prevent possible head-movement artifacts, the subject's head was firmly fixed with a strap across the subject's forehead that was secured by calipers built into the head coil. The stimuli were shown to the subject through an LCD projector on the RF coil inside the gantry. The blood-oxygen-level-dependent (BOLD) technique using the echo planar imaging (EPI) sequence was applied during fMRI scanning. The thickness of each image slice was $5 \mathrm{~mm}$, and no gap was allowed between the slices. The other MR parameters were: $\mathrm{TR}=3,000 \mathrm{~ms}, \mathrm{TE}=35 \mathrm{~ms}$, flip angle $=80^{\circ}$, field of view $=220 \times 220 \mathrm{~mm}$ and matrix $=64 \times 64$. 30 slices were obtained through the axial section image. The total duration of fMRI scanning was $6 \mathrm{~min}$ and $15 \mathrm{sec}$, so the number of EPI volumes per each condition was 60 . Furthermore, the MRI parameters in the T1 anatomical scan were: $\mathrm{TR}=2,800 \mathrm{~ms}, \mathrm{TE}=16 \mathrm{~ms}$, flip angle $=80^{\circ}$, field of view $=192 \times$ $220 \mathrm{~mm}$ and matrix $=192 \times 256$.

The imaging data were analyzed using SPM2 (Wellcome Department of Cognitive Neurology, London, UK) software, and a general linear model was applied. For motion correction and co-registration and to identify the anatomical location of the functional image, the brain imaging data obtained were analyzed using normalization and smoothing processes that matched a standard brain template to the image data from the experiment. The size of the smoothing kernel was 7 $\mathrm{mm}$.

In this study, the whole brain analysis was first conducted to investigate the regions of brain activation. Clusters that passed the voxel-level uncorrected threshold of $\mathrm{p}<0.001$ and an extent threshold of 5 contiguous voxels in size were considered activation regions. After conducting an individual level analysis, one-sample t-test was conducted for the group analysis. In other words, the relative activation of each region was examined for the remaining 3 conditions (PF, SR, MR) using NR as the control condition (PF vs. NR, SR vs. NR, MR vs. NR). Then, within-subject ANOVA for the brain activa- tion results from the PF, SR and MR conditions was conducted to investigate which regions showed significant differences in activation during the 3 conditions.

Moreover, the regions that were significantly activated in the PF vs. NR, SR vs. NR and MR vs. NR were selected as the regions of interest (ROI). The effective values (5 mm radius) were extracted from the normalizing image using the MarsBaR (http://marsbar.sourceforge.net) toolbox. We extracted the beta values for each condition from the individual ROIs and then calculated the value of each condition. We ran a correlation analysis to examine the relationship between the CDI or CNS scores and the extent of brain activation in each ROI.

\section{Other statistical analyses}

The response accuracy and the average reaction time for NR, PF, SR and MR were tested with repeated measures of ANOVA. The correlation between the subjects' CDI or CNS scale scores and ROI activation was analyzed using Spearman's correlation analysis with Bonferroni correction. SPSS $12.0 \mathrm{~K}$ for Windows was used for the statistical analyses. Because the number of the ROI was different in each condition, the significance levels according to Bonferroni correction were as follows; 1) in PF vs. NR, $\mathrm{p}<0.005,2)$ in SR vs. NR, $\mathrm{p}<0.004,3)$ in MR vs. NR, $\mathrm{p}<0.005$.

\section{RESULTS}

\section{Age, IQ, and psychological characteristics of the subjects}

The age distribution of all 15 participants was as follows; 1 ) age of $13, n=6,2$ ) age of $14, n=7,3$ ) age of $15, n=2$. The average age of the subjects was $13.80 \pm 0.86$ years, and their average IQ was $108.93 \pm 14.01$. All of the subjects were right-handed, and none of the subjects were smokers or currently taking any medications. In addition, there was no case that exceeded the clear clinical diagnostic threshold of K-SADS-PL-K.

The CDI score for the subjects was $8.93 \pm 4.38$ points, with 17 points being the highest CDI score among the subjects. None of the subjects scored more than 21 points, which is the cutoff point for suspecting clinical depression in Korean students.

The total score for the CNS was $109.73 \pm 26.20$ points, with factor $1=20.87 \pm 6.92$, factor $2=26.87 \pm 6.40$, factor $3=18.87 \pm$ 5.43 , factor $4=23.00 \pm 6.50$ and factor $5=20.13 \pm 6.85$.

\section{Comparison of response accuracy and reaction time among each of 4 conditions}

The accuracy for the right-left discrimination test was found to be as follows: NR 99.00\%, PF 99.67\%, SR 100\% and MR 99.67\%. No statistically significant differences were found 
between the accuracy for each condition $(\mathrm{F}=0.189, \mathrm{df}=3$, $\mathrm{p}=0.903)$. And, the subjects' reaction times were $179.67 \pm$ $67.95 \mathrm{msec}$ for the NR condition, $175.60 \pm 57.49 \mathrm{msec}$ for the PF condition, $175.00 \pm 63.97 \mathrm{msec}$ for the SR condition and $163.33 \pm 51.19 \mathrm{msec}$ for the MR condition. No statistically significant differences in reaction time were identified by the ANOVA test $(\mathrm{F}=1.876, \mathrm{df}=3, \mathrm{p}=0.148)$.

\section{Results of fMRI data analyses}

Regions that were activated significantly more in each reward condition compared to the NR condition

(Table 1, Figure 1)

The left superior frontal gyrus (BA 9), the right caudate body and the right ventral anterior nucleus of the thalamus were activated significantly more in the PF condition than the NR condition. The right middle frontal gyrus (BA 46), the right postcentral gyrus (BA 3), the right culmen of the cerebellum and the right caudate head showed significantly more activation in the SR condition than the NR condition. The left pulvinar of the thalamus, the right middle frontal gyrus (BA 10) and the right caudate head showed significantly more activation in the MR condition than the NR condition.

\section{ANOVA results for brain activation in the 3 conditions}

When the extent of brain activation in each of the three conditions (PF, SR and MR) was compared, the region that showed significant difference included the right cuneus (BA 30 ) only. In case that the cluster criteria were lowered to $\mathrm{p}<$ 0.002 and extent threshold $\mathrm{k}=5$, the regions that showed significant differences included the right precentral gyrus (BA 6), the left paracentral lobule (BA 31) and the left middle frontal gyrus (BA 46). The right cuneus showed greater activation in PF condition than in the other two conditions, whereas the activation of the right precentral gyrus and the left paracentral lobule in SR condition were significantly higher than in the other two conditions. Finally, the activation of the left middle frontal gyrus in MR condition was significantly higher than in the other two conditions (Figure 2).

\section{Correlation between the brain activity of significant regions in reward conditions and the CDI and CNS scores}

The regions that were activated significantly higher in each reward condition than in the NR condition were considered as ROI to examine for a correlation with CDI and CNS scores. There was no significant correlation between the degree of activation of the ROIs in each condition and each score.

\section{DISCUSSION}

The right caudate area was activated in all of the conditions. The caudate nucleus is a subdivision of the dorsal striatum where dopaminergic nerve terminals are closely distributed. The caudate nucleus has been reported frequently as being activated in association with monetary rewards, ${ }^{11,25}$ and a broad range of areas in the striatum, including the caudate nucleus and the putamen, have been reported to be activated in response to stimuli providing social rewards. ${ }^{9,10}$ Several studies have also reported the activation of the caudate nucleus in response to performance feedback. ${ }^{12,13}$ With reference to the reward response of adolescents, Van Leijenhorst et al. ${ }^{5}$

Table 1. Significantly activated brain regions associated with each reward vs. no reward condition (uncorrected, $p<0.001$, extent threshold $k=5$ )

\begin{tabular}{|c|c|c|c|c|c|c|c|}
\hline Location & Region & $\mathrm{X}$ & $\mathrm{Y}$ & $\mathrm{Z}$ & BA & $\mathrm{Ke}$ & $Z$ value \\
\hline \multicolumn{8}{|c|}{ PF vs. NR } \\
\hline Left & Superior frontal gyrus & -3 & 52 & 29 & 9 & 32 & 3.63 \\
\hline Right & Caudate body & 17 & 14 & 11 & N/A & 10 & 3.53 \\
\hline Right & Thalamus (VAN) & 15 & -6 & 15 & N/A & 9 & 3.46 \\
\hline \multicolumn{8}{|c|}{ SR vs. NR } \\
\hline Right & Middle frontal gyrus & 41 & 23 & 20 & 46 & 9 & 3.53 \\
\hline Right & Postcentral gyrus & 22 & -36 & 66 & 3 & 5 & 3.43 \\
\hline Right & Culmen & 4 & -45 & 0 & N/A & 10 & 3.33 \\
\hline \multirow[t]{2}{*}{ Right } & Caudate head & 6 & 4 & -2 & N/A & 15 & 3.28 \\
\hline & MR vs. NR & & & & & & \\
\hline Left & Thalamus (pulvinar) & -3 & -32 & 8 & N/A & 5 & 3.52 \\
\hline Right & Middle frontal gyrus & 34 & 36 & 21 & 10 & 6 & 3.35 \\
\hline Right & Caudate head & 5 & 6 & 0 & N/A & 6 & 3.34 \\
\hline
\end{tabular}

The statistical analyses were done by one sample t-test. Coordinates follow atlas by Talairach and Tournoux (1988). PF: performance feedback, SR: social reward, MR: monetary reward, NR: no reward, VAN: ventral anterior nucleus, BA: Brodmann area, N/A: not applicable 


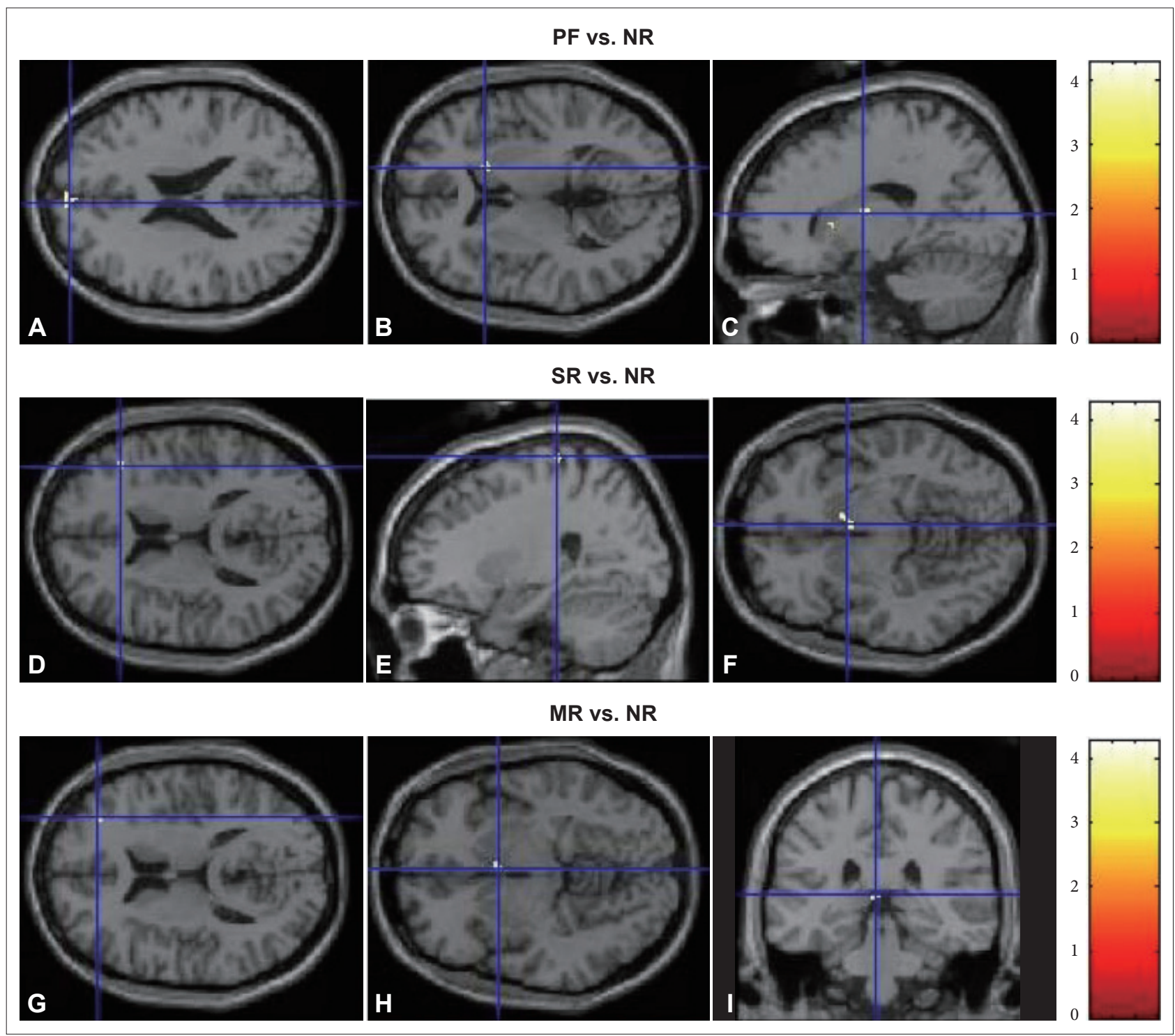

Figure 1. Significantly activated brain regions associated with each reward vs. no reward condition (uncorrected, $p<0.001$, extent threshold $\mathrm{K}=5)$. A: It. superior frontal gyrus. B: rt. caudate body. C: rt. Thalamus (VAN). D: rt. middle frontal gyrus. E: rt. postcentral gyrus. F: rt. caudate head. G: rt. middle frontal gyrus. H: rt. caudate head. I: It. thalamus. PF: performance feedback, SR: social reward, MR: monetary reward, NR: no reward, VAN: ventral anterior nucleus.

proved that the striatum showed a reward-related peak in middle adolescence (14- to 15-year-old) in anticipation of uncertain outcome. Galvan et al. ${ }^{3}$ also showed similar results. The result of our study suggest that the various rewards might activate caudate area in adolescents, especially, regardless of the type of reward.

The left superior frontal gyrus (BA 9) in PF-NR condition, the right middle frontal gyrus (BA 46) in SR-NR condition and the right middle frontal gyrus (BA 10) in MR-NR condition correspond to the dorsomedial or the dorsolateral prefrontal cortex (DLPFC). Although the BA 9 activation region in the PF-NR condition is close to the dorsomedial area, no particular difference in the function of the medial and lateral areas has been reported for the BA 9 region. Is the activation of the DLPFC area also common in the brain response to feedback and rewards? Wallis and Kennerley ${ }^{26}$ suggested that reward signals in lateral prefrontal cortex are consistent with a role in using reward to guide other cognitive processes, such as the allocation of attentional resources. They also insisted that lateral prefrontal neurons encode the association between the stimulus and response rather than information about the outcome (that is, reward itself). The studies about the DLPFC in related with reward task in adolescents were not yet reported. However, considering that the prefrontal region of brain undergo a startling transformation in adolescent period (for example, synapse pruning) and 'reward seeking behavior' is 

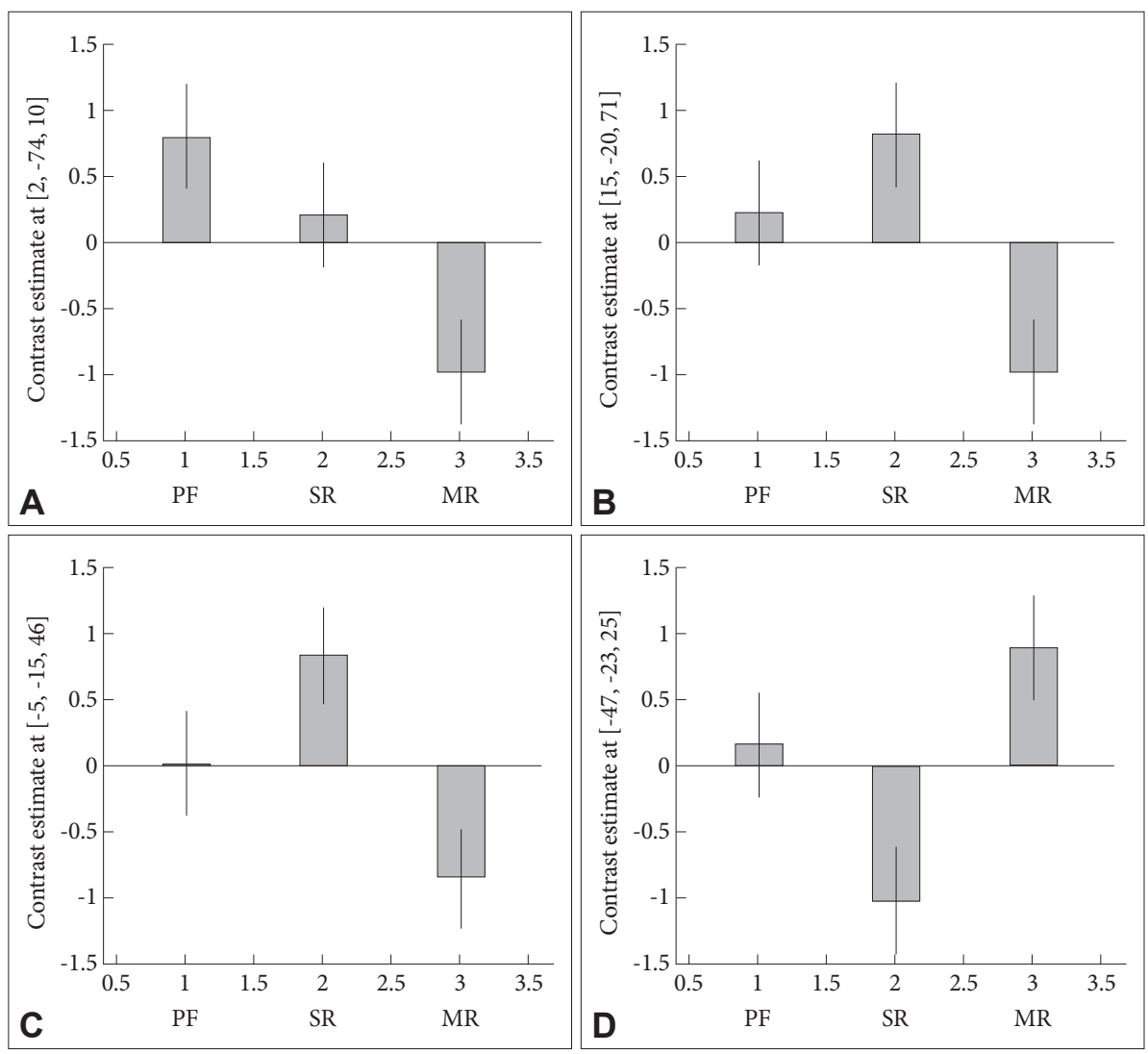

Figure 2. Contrast estimate among PF, $\mathrm{SR}$, and MR conditions (A: uncorrected, $p<0.001$, extent threshold $k=5$ ). $B-D$ : uncorrected, $p<0.002$, extent threshold $k=5$ ). A: rt. cuneus (BA 30). B: rt. precentral gyrus (BA 6). C: It. paracentral lobule (BA 31). D: It. middle frontal gyrus (BA 46). Coordinates follow atlas by Talairach and Tournoux (1988). PF: performance feedback, SR: social reward, MR: monetary reward, BA: Brodmann area.

gaining much attention to adolescents, the noticeable activation of dorsal prefrontal area for various reward tasks might easily occur in adolescents. A follow-up study to investigate this assumption seems necessary.

The thalamic area was activated in both the PF-NR and the MR-NR conditions. The thalamus is a structure that is located in the center of the brain near the caudate nucleus, accepting external stimuli and governing arousa ${ }^{27}$ rather than logical thinking. Our result suggest that the adolescents in our study might be little aroused by the social reward such as 'great' or 'good'.

The right postcentral gyrus was activated in the SR-NR condition only. Several studies in the field of social neuroscience report that this area is related to self-related processing ${ }^{28}$ or feelings about oneself. ${ }^{29}$ Such a response to the SR was also found in a previous study on adults. ${ }^{14}$ The studies about direct interaction between the SR and brain response in adolescents are not yet reported, but Steinberg ${ }^{30}$ reported the brain response on performing a driving game in adolescents. When they performed the game with peer-present condition, the medial frontal cortex, left superior temporal sulcus, and left medial temporal structure were activated. These areas are related with self-related processing or theory of mind. This report would be one of the evidence that the brains of adolescents react very sensitively to other people's assessments about them, which are similar with SR. The fact that only the right brain area was activated in the SR-NR condition may support this belief. The right brain is known to be more closely related to the self-referential function than the left brain.

Meanwhile, there were some differences in the regions that were activated for each condition when all reward conditions were compared at once.

In case of the cluster criteria of $\mathrm{p}<0.001$ and extent threshold $\mathrm{k}=5$, the $\mathrm{PF}$ condition activated the right cuneus significantly more than the other conditions. The cuneus is mainly responsible for basic visual processing, but a study found that the degree of response inhibition and the volume of the cuneus showed a positive correlation in bipolar disorder patients ${ }^{31}$ and the cuneus has even been reported as one of the regions that is also activated by feedback-related tasks. ${ }^{32}$

In case that the cluster criteria were adjusted to $\mathrm{p}<0.002$ and extent threshold $\mathrm{k}=5$, another regions that showed somewhat different activation among 3 reward conditions were found. First, the SR condition activated the right precentral gyrus and the right paracentral lobule slightly more than the other conditions. Similar with the postcentral gyrus, the right precentral gyrus is a region associated with self-related processing, and the right paracentral lobule is also part of the cortical midline structure, which is the major self-related area. The cortical midline structure was reported to be a region re- 
lated to reward outcome rather than reward anticipation. ${ }^{33}$ Second, the MR condition activated the left middle frontal gyrus slightly more than the other conditions. This region corresponds to the dorsolateral prefrontal area. Considering that the the right middle frontal gyrus in the MR vs. NR condition also showed significant activation in within-subject analysis, the activation of bilateral dorsal prefrontal area for maintaining attentional resources would be very important for performing monetary-related task in adolescents.

Finally, the correlation between the extent of activation in each condition and the psychological characteristics was not significant. If the general threshold (that is, $\mathrm{p}<0.05$ ) without Bonferroni correction was adopted, there was a considerable result between the degree of right caudate activation and CDI score in the MR conditions ( $\mathrm{r}=0.555, \mathrm{p}=0.332$ ). However, according to Fobes et al., ${ }^{34}$ the MR-mediated activation of the reward-related brain region was lower in depressed adolescents than in normal adolescents. The fact that the CDI scores in our study were all below the clinical cutoff point, which is different from the study of Fobes et al., can suggest one important idea. That is, the increase of the depressive feeling in normal adolescents make their brain respond more sensitively to monetary rewards. But, once the depressive feeling develops into major depressive disorder, then the brain responses related to monetary reward might decrease. A follow-up study to investigate this assumption seems necessary.

The limitations of this study are as follows.

First, the total duration of fMRI scanning was slightly short. If we chose the paradigm that included longer task, the statistical power of our results would be higher. Second, the sample size was small, which limits the comparison of various psychological test scales and the extent of brain activation. Third, because this study was conducted with block-based design, we were unable to observe more detailed brain activation for reward anticipation or reward outcome. Fourth, the fact that the subjects of this study were early adolescents must be taken into consideration.

Despite the above limitations, this study is meaningful in that it examined various responses to rewards and feedback in adolescents. Not only various reward stimuli but also feedback stimulus might commonly activate the caudate nucleus and the dorsal prefrontal area in adolescents. The results of this study can be applied to planning of adolescent learning and teaching at home or school in various ways. Furthermore, these results are also expected to be one of the basic reference of brain studies investigating reward responses for adolescents with psychopathology. We look forward to more sophisticated reward-related brain research on adolescents in the future.

\section{Acknowledgments}

The authors gratefully acknowledge the assistance of all persons and volunteers whose participation was essential in the successful completion of the study.

\section{REFERENCES}

1. Galvan A. Adolescent development of the reward system. Front Hum Neurosci 2010;4:6.

2. Kelley AE, Schochef T, Landry CF. Risk taking and novelty seeking in adolescence: introduction to part 1. Ann N Y Acad Sci 2004;1021:2732.

3. Galvan A, Hare TA, Parra CE, Penn J, Voss H, Glover G, et al. Earlier development of the accumbens relative to orbitofrontal cortex might underlie risk-taking behavior in adolescents. J Neurosci 2006;26:68856892.

4. Bjork JM, Knutson B, Fong GW, Caggiano DM, Bennett SM, Hommer DW. Incentive-elicited brain activation in adolescents: similarities and differences from young adults. J Neurosci 2004;24:1793-1802.

5. Van Leijenhorst L, Zanolie K, Van Meel CS, Westenberg PM, Rombouts SA, Crone EA. What motivates the adolescent? Brain regions mediating reward sensitivity across adolescence. Cereb Cortex 2010;20: 61-69.

6. Knutson B, Fong GW, Adams CM, Varner JL, Hommer D. Dissociation of reward anticipation and outcome with event-related fMRI. Neuroreport 2001;12:3683-3687.

7. Kirsch P, Schienle A, Stark R, Sammer G, Blecker C, Walter B, et al. Anticipation of reward in a nonaversive differential conditioning paradigm and the brain reward system: an event-related fMRI study. Neuroimage 2003;20:1086-1095.

8. Di Chiara G, Bassareo V. Reward system and addiction: what dopamine does and doesn't do. Curr Opin Pharmacol 2007;7:69-76.

9. Izuma K, Saito DN, Sadato N. Processing of social and monetary rewards in the human striatum. Neuron 2008;58:284-294.

10. Zink CF, Tong Y, Chen Q, Bassett DS, Stein JL, Meyer-Lindenberg A. Know your place: neural processing of social hierarchy in humans. Neuron 2008;58:273-283.

11. Spreckelmeyer KN, Krach S, Kohls G, Rademacher L, Irmak A, Konrad $\mathrm{K}$, et al. Anticipation of monetary and social reward differently activates mesolimbic brain structures in men and women. Soc Cogn Affect Neurosci 2009;4:158-165.

12. Tricomi E, Delgardo MR, McCandliss BD, McClelland JL, Fiez JA. Performance feedback drives caudate activation in a phonological learning task. J Cogn Neurosci 2006;18:1029-1043.

13. Nieuwenhuis S, Slagter HA, von Geusau NJ, Heslenfeld DJ, Holroyd CB. Knowing good from bad: differential activation of human cortical areas by positive and negative outcomes. Eur J Neurosci 2005;21:31613168.

14. Pan X, Hu Y, Li L, Li J. Evaluative-feedback stimuli selectively activate the self-related brain area: an fMRI study. Neurosci Lett 2009;465:9094.

15. Pizzagalli DA, Iosifescu D, Hallett LA, Ratner KG, Fava M. Reduced hedonic capacity in major depressive disorder evidence from a probabilistic reward task. J Psychiatr Res 2008;43:76-87.

16. Forbes EE, May JC, Siegle GJ, Ladouceur CD, Ryan ND, Carter CS, et al. Reward-related decision-making in pediatric major depressive disorder: an fMRI study. J Child Psychol Psychiatry 2007;47:1031-1040.

17. Cooper A. Further Developments in the Clinical Diagnosis of Narcissistic Personality Disorder, Disorder of Narcissism. Diagnostic, Clinical and Empirical Implication. London: Jason Aronson Inc.; 2000.

18. Kim YS, Cheon KA, Kim BN, Chang SA, Yoo HJ, Kim JW. The reliability and validity of Kiddie-Schedule for Affective Disorders and Schizophrenia for School-Age Children-Present and Lifetime Version-Korean Version. Yonsei Med J 2004;45:81-89.

19. Kaufman AS. A four-test short form of the WISC-R. Contemp Educ 
Psychol 1976;1:180-196.

20. Cho SC, Lee YS. Development of the Korean form of the Kovacs' Children's Depression Inventory. J Korean Neuropsychiatr Assoc 1990;29: 945-956.

21. Kovacs M. The Children's Depression Inventory: A Self-rated Depression Scale for School Aged Youngsters. Pittshburgh : University of Pittshburgh; 1983.

22. Gang SH, Chung NW. A study on the development and validation of the Covert Narcissism Scale. Korean J Couns Psychol 2002;14:969-990.

23. Akhtar S, Thomson JA Jr. Overview: narcissistic personality disorder. Am J Psychiatry 1982;139:12-20.

24. Ghandi SP, Heeger DJ, Boynton GM. Spatial attention affects brain activity in human primary visual cortex. Proc Natl Acad Sci USA 1999;96: 3314-3319.

25. Gotlib IH, Hamilton JP, Cooney RE, Singh MK, Henry ML, Joormann J. Neural processing of reward and loss in girls at risk for major depression. Arch Gen Psychiatry 2010;67:380-387.

26. Wallis JD, Kennerley SW. Heterogeneous reward signals in prefrontal cortex. Curr Opin Neurobiol 2010;20:191-198.

27. Packard MG, Knowlton BJ. Learning and memory functions of the
Basal Ganglia. Annu Rev Neurosci 2002;25:563-593.

28. Ruby P, Decety J. What you believe versus what you think they believe: a neuroimaging study of conceptual perceptive taking. Eur J Neurosci 2003;17:2475-2480.

29. Kircher TTG, Brammer M, Bullmore E, Simmons A, Bartels M, David AS. The neural correlates of intentional and incidental self processing. Neuropsychologia 2002;40:683-692.

30. Steinberg L. A social neuroscience perspective on adolescent risk-taking . Dev Rev 2008;28:78-106.

31. Haldane M, Cunningham G, Androutsos C, Frangou S. Structural brain correlates of response inhibition in Bipolar Disorder I. J Psychopharmacol 2008;22:138-143.

32. Volz KG, Schubotz RI, von Cramon DY. Frontomedian activation depends on both feedback validity and valence: fMRI evidence for contextual feedback evaluation. Neuroimage 2005;27:564-571.

33. Berridge KC, Robinson TE. Parsing reward. Trend Neurosci 2003;26: 507-513.

34. Forbes EE, Hariri AR, Martin SL, Silk JS, Moyles DL, Fisher PM, et al. Altered striatal activation predicting real-world positive affect in adolescent major depressive disorder. Am J Psychiatry 2009;66:64-73. 